\title{
Real-World Analysis of Therapeutic Patterns in Patients Affected by Rheumatoid Arthritis in Italy: A Focus on Baricitinib
}

\author{
Valentina Perrone (D) - Serena Losi · Veronica Rogai · Silvia Antonelli • \\ Walid Fakhouri · Massimo Giovannitti · Elisa Giacomini · \\ Diego Sangiorgi $\cdot$ Luca Degli Esposti
}

Received: April 29, 2020 / Published online: July 18, 2020

(C) The Author(s) 2020

\section{ABSTRACT}

Introduction: The objective of this study was to evaluate treatment patterns in patients with rheumatoid arthritis (RA), with a focus on the utilization of baricitinib, an oral highly selective Janus kinase 1 and 2 inhibitor, in an Italian realworld setting.

Methods: This observational retrospective analysis was based on data collected in selected Italian administrative databases. Patients aged $\geq 18$ years with a diagnosis of RA defined by hospitalization discharge diagnosis

Digital Features To view digital features for this article go to: https://doi.org/10.6084/m9.figshare.12554675.

Electronic Supplementary Material The online version of this article (https://doi.org/10.1007/s40744020-00218-3) contains supplementary material, which is available to authorized users.

V. Perrone $(\bowtie) \cdot$ E. Giacomini · D. Sangiorgi · L. Degli Esposti

Clicon S.r.l. Health Economics and Outcomes Research, Ravenna, Italy

e-mail: valentina.perrone@clicon.it

S. Losi - V. Rogai · S. Antonelli

Eli Lilly Italy S.p.A., Sesto Fiorentino, Italy

W. Fakhouri

Eli Lilly and Company Limited, Windlesham, Surrey, UK

M. Giovannitti

Eli Lilly Italy S.p.A., Rome, Italy
(International Classification of Diseases, Ninth Revision, Clinical Modification code 714.0) or by disease exemption code 006 for RA in 2018 were included. The index date (ID) was defined as the date of first prescription for a drug indicated for RA during the inclusion period. Patients without a prescription for biologic/targeted synthetic disease-modifying antirheumatic drugs (b/tsDMARDs) before the ID were considered to be $b / t s D M A R D$ naïve. A further analysis was performed on patients only receiving baricitinib.

Results: A total of 41,290 RA patients were enrolled, of whom $55.6 \%$ were not treated with conventional synthetic DMARDs (csDMARDs) or b/tsDMARDs, $39.4 \%$ were receiving therapy with csDMARDs, and $5.0 \%$ were using b/tsDMARDs. In the latter group, $2.7 \%(n=56)$ were receiving therapy with baricitinib. In 2018, $13.2 \%$ of csDMARD-treated patients switched to $\mathrm{b} /$ tsDMARDs, of whom $4.3 \%(n=93)$ of these switched to baricitinib. In total, 149 patients (mean age \pm standard deviation $57.6 \pm 12.1$; $12.8 \%$ male) had a baricitinib prescription, of whom 51\% were b/tsDMARD naïve. At baseline, $61.7 \%$ of baricitinib users were receiving combination therapy with csDMARDs plus corticosteroids, $26.2 \%$ were receiving combination therapy with corticosteroids, and $8.1 \%$ were receiving combination therapy with csDMARDs; $4 \%$ were receiving baricitinib monotherapy. During follow-up, the proportion of patients receiving baricitinib 
monotherapy increased to $38.9 \%$, while 26.9 , 18.8 , and $15.4 \%$ of baricitinib users received combination therapy with corticosteroids, csDMARDs plus corticosteroids, and csDMARDs, respectively.

Conclusion: This study provides a current view of the treatment patterns in Italian patients with RA in a real-world setting of daily clinical practice, with a focus on baricitinib utilization.

Keywords: Baricitinib; Biologic DMARDs; Realworld study; Rheumatoid arthritis; Targeted synthetic DMARDs; Treatment patterns

\section{Key Summary Points}

The field of therapeutic options for rheumatoid arthritis (RA) is growing rapidly and calls for more evidence from routine clinical practice to assess the prescription patterns in real-world rheumatology practice.

An in-depth analysis focused on baricitinib utilization is provided in an Italian real-world setting.

More than one-half of the patients affected by RA screened did not receive any form of disease-modifying antirheumatic drugs (DMARDs), either conventional synthetic, targeted synthetic (ts), or biologic (b).

Approximately one-half of patients treated with baricitinib were naïve to b/tsDMARDs.

\section{INTRODUCTION}

Rheumatoid arthritis (RA) is one of the most common chronic inflammatory diseases and is characterized by the inflammation of the synovial membranes, causing chronic pain, swelling, and stiffness in the joints $[1,2]$.

If left untreated, RA can lead to loss of physical function caused by joint destruction; therefore, an early initiation of RA therapy upon diagnosis is required to achieve optimal outcomes, such as persistent low disease activity or remission [3]. Disease-modifying antirheumatic drugs (DMARDs) are the mainstay of RA therapy. Both the Italian Society for Rheumatology clinical practice guidelines [4] and the most updated published European League Against Rheumatism (EULAR) guidelines [5] for RA recommend the adoption of conventional synthetic DMARDs (csDMARDs) as initial therapy, with methotrexate considered to be the "anchor drug" as monotherapy in the first-line treatment strategy. According to the Italian guidelines, the csDMARDs leflunomide and sulfasalazine can be administered as a first-line therapy in patients with contraindications for methotrexate. In these initial treatment steps, the concomitant use of short-term corticosteroids (CS) is advised when initiating or changing csDMARDs. If the treatment target, i.e., sustained remission or low disease activity, has not been achieved with csDMARDs, in the absence of poor prognostic factors a switch to a second csDMARD or to a combination of two csDMARDs should be considered. However, if prognostically unfavorable factors are present, current recommendations are to add a biological DMARD (bDMARD) or a targeted synthetic DMARD (tsDMARD) to the ongoing treatment. When treatments with $\mathrm{b} / \mathrm{tsDMARDs}$ have failed, changing to other agents with the same or different modes of action are recommended.

Among the bDMARDs currently available are numerous drugs with different mechanisms of action, including tumor necrosis factor inhibitors, interleukin blockers, T-cell costimulation modulators, and anti-B-cell agents. Recently, the Janus kinase (JAK) inhibitors tofacitinib and baricitinib became the first tsDMARDs to be approved for RA [6, 7].

The field of therapeutic options for RA management is expanding rapidly, and this rapid expansion calls for more evidence from routine clinical practice to evaluate the longterm effectiveness of any given therapy as well as to assess the prescription patterns in realworld rheumatology practice. In this context, the aim of the present study was to describe treatment patterns in patients with RA based on 
the most updated available data in an Italian real-world setting, with an in-depth analysis focused on baricitinib utilization. Baricitinib has been approved for reimbursement by the Ministry of Health in Italy since 2017 for the treatment of patients with moderate to severe RA, either as monotherapy or in combination with csDMARDs [8].

\section{METHODS}

\section{Data Sources}

This observational study was based on data collected in administrative databases of selected Italian settings, including approximately 12 million health-assisted individuals, representing approximately $20 \%$ of the Italian population. The following databases were used in the analyses: the 'beneficiaries' database' that contains patients' demographic data; the 'pharmaceutical databases' (inpatient and outpatient) that provide data on prescriptions, such as Anatomical-Therapeutic-Chemical (ATC) codes, the number of packages, the number of units per package, and the prescription date; the 'hospitalization database' that includes all hospitalization data with discharge diagnosis codes classified according to the International Classification of Diseases, Ninth Revision, Clinical Modification (ICD-9-CM); and the 'exemption ticket for pathology database' that includes disease exemption codes and the dates of exemption.

To guarantee patients' privacy, an anonymous univocal numeric code was assigned to each subject included in the study, in full compliance with the European General Data Protection Regulation (GDPR) (2016/679). In each database, this code allowed for the electronic linkage of all the databases. No identifiers related to patients were provided to the authors. All the results of the analyses were produced as aggregated summaries, which are not possible to assign, either directly or indirectly, to individual patients.

Informed consent was not required for the use of this encrypted retrospective information for research purposes. In accordance with
Italian law regarding the performance of observational analysis [9], the Ethics Committee of each participating entity (Supplementary Material) was notified of this study, and the relevant Ethics Committees approved the study.

\section{Study Population}

All patients aged $\geq 18$ years were included in the study if they had received a diagnosis of RA identified by at least one hospitalization with a relevant primary/secondary discharge diagnosis (ICD-9-CM code 714.0) or at least an exemption code (006.714.0) during 2018 (inclusion period). The date of the first prescription for a drug indicated for the treatment of RA during the inclusion period was considered the index date (ID). Patients were characterized the year before the ID (characterization period) and followedup from the ID to the end of the study. An analysis that focused on baricitinib users was performed, including only patients who received a prescription for baricitinib during the inclusion period.

Patients without a prescription for $\mathrm{b} /$ tsDMARDs before the ID were considered to be "b/tsDMARDs naïve," while those who had received at least one prescription for such drugs before the ID were regarded as "established."

\section{Study Variables}

At baseline, data on demographic characteristics such as age and sex were collected.

The following therapies were analyzed to assess treatment patterns: csDMARDs [methotrexate (ATC L04AX03), ciclosporin (ATC L04AD01), sulfasalazine (ATC A07EC01), leflunomide (ATC L04AA13), hydroxychloroquine (ATC P01BA02)], CS (ATC H02), nonsteroidal anti-inflammatory drugs (NSAIDs) (ATC M01), bDMARDs [abatacept (ATC L04AA24), adalimumab (ATC L04AB04), anakinra (ATC L04AC03), canakinumab (ATC L04AC08), certolizumab (ATC L04AB05), etanercept (ATC L04AB01), golimumab (ATC L04AB06), infliximab (ATC L04AB02), rituximab (ATC L01XC02), tocilizumab (ATC 


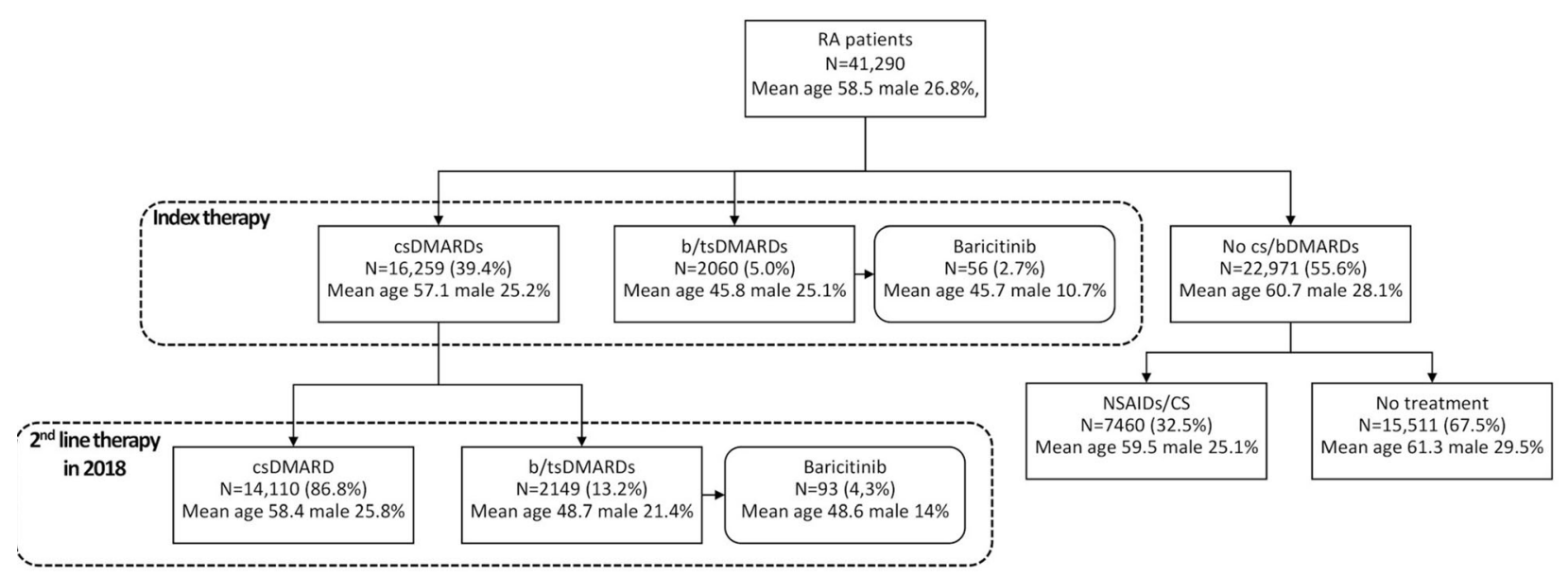

Fig. 1 Flow chart of the patients with rheumatoid arthritis $(R A)$ stratified according to the pattern of treatment. csDMARDs or $b / t s D M A R D s$ Conventional synthetic or biologic/targeted synthetic disease-modifying antirheumatic

L04AC07)], and tsDMARDs [baricitinib (ATC L04AA37)].

\section{Statistical Analysis}

All analyses were descriptive. Continuous variables are reported as the means \pm standard deviations (SD), whereas categorical variables are expressed as frequencies and percentages. All analyses were performed using the STATA SE version 12.0 statistical software (StataCorp LP, College Station, TX, USA).

\section{RESULTS}

Overall, 41,290 patients were included in the study. The mean age of the patients was 58.5 years, and $26.8 \%$ were male. The distribution of patients stratified according to the pattern of treatment is shown in Fig. 1. As index therapy, 16,259 (39.4\%) patients were prescribed csDMARDs, and 2060 (5\%) were prescribed b/tsDMARDs; in this latter group, 56 $(2.7 \%)$ patients were treated with baricitinib. Among the 22,971 (55.6\%) patients without cs/ b/tsDMARD prescriptions, only 7460 (32.5\%) were treated with NSAIDs and/or CS, while the remaining had no treatment indicated for RA. drugs, NSAIDs nonsteroidal anti-inflammatory drugs, CS corticosteroids

In the cohort of patients receiving csDMARD treatment as index therapy, in 2018, 14,110 $(86.8 \%)$ continued with conventional therapies, while $2149(13.2 \%)$ changed to b/tsDMARDs as a second-line therapy, of whom 93 (4.3\%) had a prescription for baricitinib.

Considering both the index and second-line therapy, a total of 149 patients were treated with baricitinib and followed-up for a mean (SD) of 103 (46) days. The mean age was 57.6 years, and the proportion of males was $12.8 \%$.

The co-presence of prescriptions for conventional therapies in baricitinib users as well as in patients treated with bDMARDs was investigated. At baseline, 61.7 and $36.1 \%$ of patients in the baricitinib (Fig. 2a) and bDMARDs (Fig. 2b) cohorts, respectively, were co-treated with csDMARDs plus CS, 8.1 and $22.1 \%$, respectively, were co-treated with csDMARDs, and 26.2 and $20.5 \%$, respectively, were co-treated with CS. Patients not co-treated with csDMARDs and/or CS accounted for $4.0 \%$ of the baricitinib group and $21.3 \%$ of the bDMARD cohort.

During follow-up, the percentage of patients in the baricitinib group co-treated with csDMARDs and CS dropped to $18.8 \%$, the percentage of those treated with combination therapy with csDMARDs increased to $15.4 \%$, and the percentage prescribed CS remained 
almost the same (26.9\%) (Fig. 3a). In the bDMARD cohort, during a mean (SD) follow-up of 201 (65) days after the first prescription for bDMARDs, the percentage of patients co-treated with csDMARDs plus CS decreased to $22.1 \%$, the percentage of patients co-treated with csDMARDs slightly increased to $23.9 \%$, and the percentage of patients co-treated with CS remained almost unchanged (19.7\%) (Fig. 3b). The proportion of patients receiving monotherapy, i.e., without a prescription for csDMARDs and/or CS, increased from 4.0 to $38.9 \%$ in the baricitinib group and from 21.3 to $34.3 \%$ in the bDMARD cohorts.

Among baricitinib-treated patients, in the available periods before the ID, 76 (51.0\%) patients were $\mathrm{b} / \mathrm{tsDMARD}$ naïve, 33 (22.1\%) had at least one previous bDMARD prescription, and $28(18.8 \%)$ and $12(8.1 \%)$ were previously prescribed two and three or more bDMARDs, respectively. Among b/tsDMARD naïve patients, during follow-up, $31.6 \%$ received baricitinib as combination therapy, while $68.4 \%$ received it as a monotherapy. During the characterization period, $16.1 \%$ of baricitinib patients were previously treated with tocilizumab, $14.8 \%$ with abatacept, $12.1 \%$ with etanercept, $5.4 \%$ with golimumab, $4.7 \%$ with adalimumab, and $4.0 \%$ with certolizumab.

\section{DISCUSSION}

In the present study, we assessed the treatment patterns among adult patients diagnosed with RA in an Italian real-world setting, with a focus on baricitinib utilization among patients with RA in Italy. The most updated data from routine a. Baricitinib patients $(\mathrm{N}=149)$

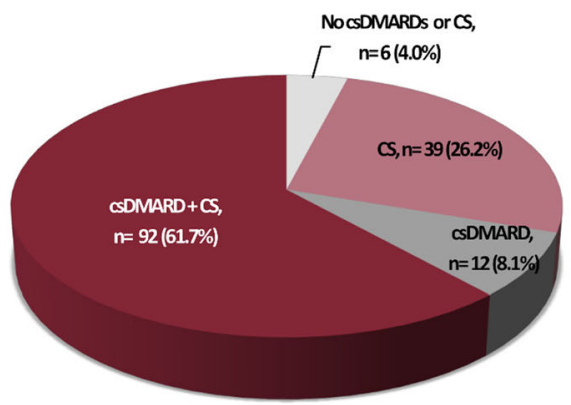

b. bDMARDs patients $(\mathrm{N}=4060)$

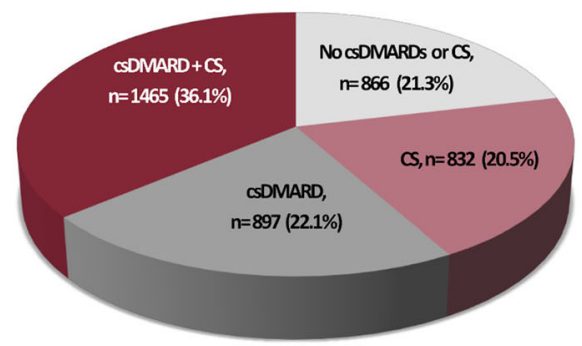

Fig. 2 Co-treatment patterns at baseline in patients receiving baricitinib (a) and bDMARDs (b). csDMARDs conventional synthetic disease-modifying antirheumatic drugs, $C S$ corticosteroids
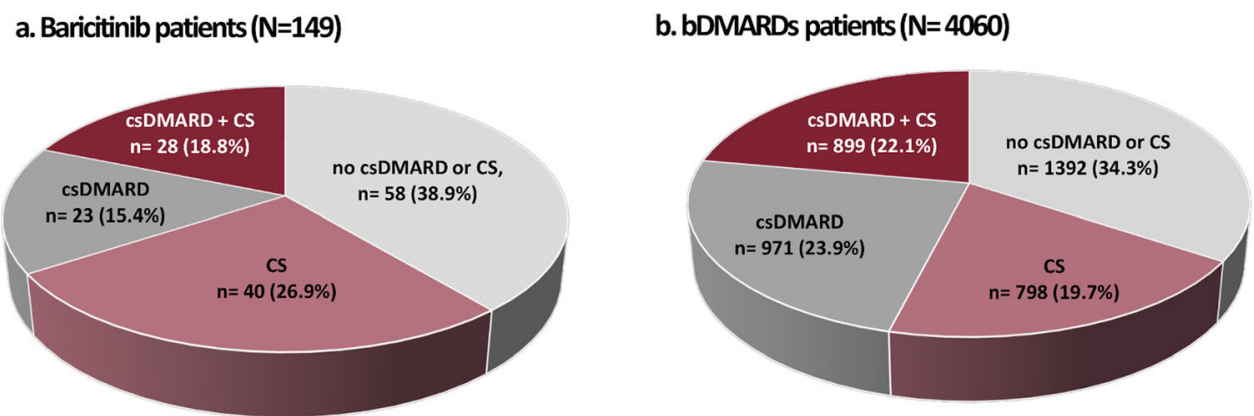

Fig. 3 Co-treatment patterns during follow-up in patients receiving baricitinib (a) and bDMARDs (b). csDMARDs conventional synthetic disease-modifying antirheumatic drugs, $C S$ corticosteroids 
clinical practice were used in the analysis. This is the first report on baricitinib utilization in this patient group.

Our results show that approximately onehalf of the patients were receiving DMARD therapy, with $b / t s D M A R D s$ accounting for only $5 \%$ of prescriptions as index therapy and for $13.2 \%$ of prescription as second-line therapy after csDMARD treatment. Because administrative databases do not collect data on the severity of disease, according to the guidelines in force during the study period [10], the patients included may either have achieved a low disease activity state/remission or be still on csDMARDs, despite not yet reaching positive outcomes. Similarly, in another Italian realworld study conducted by Fakhouri et al. [11] involving patients with RA undergoing therapy, the majority of the patients were receiving csDMARDs, and approximately 3\% were prescribed a biologic agent as index therapy. Steffen et al. [12] found a similar pattern of DMARD prescriptions among newly diagnosed patients with RA in an ambulatory setting in Germany; over the first year of the disease, $41 \%$ of patients received csDMARDs and $3.3 \%$ received bDMARDs (no tsDMARDs were available during the study period), while in contrast with our results, approximately $70 \%$ of patients without DMARD prescriptions were treated with NSAIDs and/or CS.

Regarding co-treatment patterns of bDMARDs and baricitinib, our findings showed that the use of a monotherapy regimen increased during follow-up, being observed in approximately one-third of patients. Despite the guidelines recommending, when possible, the administration of a bDMARD in combination with csDMARDs and clinical trials supporting the superiority of the combination bDMARD and csDMARDs, real-world data from European and USA registries on the use of bDMARDs show that monotherapy is observed in approximately $30 \%$ of patients with RA $[13,14]$.

Over the past decades, the availability of bDMARDs has represented a large step forward in the treatment of patients with chronic autoimmune rheumatic diseases, such as RA, who require long-life therapy. Such drugs have improved the quality of life and reduced the disability and mortality of these patients [15]. Baricitinib, a small synthetic molecule available as an oral formulation, was the first tsDMARD to be approved, very recently, for patients affected by moderate to severe RA, and it can be administered alone or in combination with conventional therapies. To date, several studies have been published on the clinical efficacy and safety of baricitinib [16, 17], whereas almost no evidence is available its utilization in clinical practice in Italy. The aim of the present study was to fill this gap by focusing on baricitinib usage during the first year after reimbursement approval by the Ministry of Health. Our results indicate that baricitinib users were mainly women, with a mean age of 57 years. The adoption of a monotherapy regimen increased during follow-up. Approximately half of patients treated with baricitinib were b/tsDMARD naïve, and among this group, the majority received monotherapy during followup. Baricitinib was prescribed after one previous bDMARD in approximately one-fifth of patients, while it was started after two or more bDMARDs in approximately one-fourth of patients. To the best of our knowledge, only one other observational analysis based on real-life data in Italy has been conducted to date; this study included 150 patients with RA treated with baricitinib and has been published as an abstract [18]. The demographic characteristics in that study were similar to those reported in our cohort. With regard to the patterns of treatment, in contrast to our results, the authors found a lower number $(n=57)$ of patients starting baricitinib prior to biologic agents, and among established patients, baricitinib was mostly prescribed as a fourth or higher line of therapy; however, it should be noted that more than half of the patients analyzed in that cohort had severe RA, which could explain the different pattern observed. Baricitinib utilization was also investigated in a UK study performed by Page et al. [19], with patients treated with JAK inhibitors. In contrast to our analysis, in that cohort, $28 \%$ of baricitinib users were naïve to bDMARDs, and a similar percentage was found in another British study [20], in which $70 \%$ of patients with RA treated with baricitinib had 
experienced treatment failure with at least one previous bDMARD. In this latter study, the percentage of monotherapy prescriptions of baricitinib was $36.9 \%$, which was similar to our findings (38.9\%).

We acknowledge some limitations to our study. Our cohort of patients reflected patients in real clinical practice, and the results must be interpreted while taking into account the limitations related to the observational nature of the study, which was based on data collected through administrative databases. As mentioned above, one limitation was the lack of clinical information related to the severity of RA disease in terms of disease state and the progression of the disease, comorbidities, and other potential confounders that could have influenced our results. Therefore, it was not possible to collect data on the activity of RA for each patient or to collect information related to the choice of biological agents over conventional therapies. Moreover, as data on the use of pharmacological treatments were retrieved from medical prescriptions and dispensing databases, it was not possible to track the reasons underlying the choice of co-treatment or monotherapy, and a selection bias may have occurred. Ultimately, the results of this study are limited to the population analyzed and may not be applicable to the general population. More robust data on the use of baricitinib with a longer follow-up and a larger sample size will become available in the future.

\section{CONCLUSIONS}

This study provided an updated picture of the treatment patterns of patients with RA in an Italian real-world setting of everyday clinical practice. Our results show that more than onehalf of the patients included in the study did not receive $c s / b / t s D M A R D s$ and that the use of monotherapy regimens increased during follow-up among patients prescribed bDMARDs or baricitinib. Moreover, a focus on baricitinib utilization in the first year after the Ministry of Health reimbursement approval has been included in this study for the first time, showing that $51 \%$ of patients treated with baricitinib were naïve to $\mathrm{b} / \mathrm{tsDMARDs}$, while $22.1 \%$ had a previous bDMARD prescription, and the remaining patients were previously prescribed two or more bDMARDs.

\section{ACKNOWLEDGEMENTS}

Funding. Eli Lilly purchased the study report that is the basis for this article. This article was developed with Eli Lilly and CliCon S.r.l. The views expressed here are those of the authors and not necessarily those of the financial supporters. The agreement signed by Clicon S.r.l. and Eli Lilly does not create any entityship, joint venture, or any similar relationship between parties. Clicon S.r.l. is an independent company. Neither CliCon S.r.l. nor any of their representatives are employees of Eli Lilly for any purpose. Eli Lilly is funding the journal's Rapid Service Fee.

Editorial Assistance. The manuscript was edited for English language, grammar, punctuation, spelling, and overall style by Dr. Mary A. of American Journal Experts. Eli Lilly provided funding for the assistance.

Authorship. All named authors meet the International Committee of Medical Journal Editors (ICMJE) criteria for authorship for this article, take responsibility for the integrity of the work as a whole, and have given their approval for this version to be published.

Disclosures. Valentina Perrone, Elisa Giacomini, Diego Sangiorgi and Luca Degli Esposti have nothing to disclose. Walid Fakhouri is an employee and minor stockholder of Eli Lilly \& Company Limited, UK; Massimo Giovannitti is an employee and minor stockholder of Eli Lilly S.p.a. \& Company Roma, Italy; Serena Losi, Veronica Rogai, and Silvia Antonelli are employees and minor stockholders of Eli Lilly Italy S.p.a., Italy.

Compliance with Ethics Guidelines. Informed consent was not required for the use of this encrypted retrospective information for 
research purposes. In accordance with Italian law regarding the performance of observational analysis [9], the Ethics Committee of each participating entity (Supplementary Material) was notified of the study, and the relevant Ethics Committees approved the study. The list of Ethics Committees is provided in Electronic Supplementary Material.

Data Availability. The datasets generated and analyzed during the current study are not publicly available due to the fact that CliCon is the only body in charge to treat and analyze the data. The data cannot be shared to third parties but are available from the corresponding author on reasonable request.

Open Access. This article is licensed under a Creative Commons Attribution-NonCommercial 4.0 International License, which permits any non-commercial use, sharing, adaptation, distribution and reproduction in any medium or format, as long as you give appropriate credit to the original author(s) and the source, provide a link to the Creative Commons licence, and indicate if changes were made. The images or other third party material in this article are included in the article's Creative Commons licence, unless indicated otherwise in a credit line to the material. If material is not included in the article's Creative Commons licence and your intended use is not permitted by statutory regulation or exceeds the permitted use, you will need to obtain permission directly from the copyright holder. To view a copy of this licence, visit http://creativecommons.org/licenses/by$\mathrm{nc} / 4.0 /$.

\section{REFERENCES}

1. Smolen JS, Aletaha D, McInnes IB. Rheumatoid arthritis. Lancet. 2016;388:2023-38.

2. Scott DL, Wolfe F, Huizinga TW. Rheumatoid arthritis. Lancet. 2010;376:1094-108.

3. Kohler BM, Gunther J, Kaudewitz D, Lorenz HM. Current therapeutic options in the treatment of rheumatoid arthritis. J Clin Med. 2019;8:E938.
4. Parisi S, Bortoluzzi A, Sebastiani GD, et al. The Italian Society for Rheumatology clinical practice guidelines for rheumatoid arthritis. Reumatismo. 2019;71(S1):22-49.

5. Smolen JS, Landewe R, Bijlsma J, et al. EULAR recommendations for the management of rheumatoid arthritis with synthetic and biological diseasemodifying antirheumatic drugs: 2019 update. Ann Rheum Dis 2020;0:1-15.

6. Markham A. Baricitinib: first global approval. Drugs. 2017;77:697-704.

7. Caporali R, Zavaglia D. Real-world experience with tofacitinib for the treatment of rheumatoid arthritis. Clin Exp Rheumatol. 2019;37:485-95.

8. Gazzetta Ufficiale. Agenzia Italiana del farmaco. 2017. https://www.gazzettaufficiale.it/atto/serie_ generale/caricaDettaglioAtto/originario?atto.data PubblicazioneGazzetta=2017-12-02\&atto.codice Redazionale $=17$ A07967\&elenco30giorni=false. Accessed 5 Sept 2019.

9. Agenzia Italiana del Farmaco (AIFA). Guideline for the classification and conduction of the observational studies on medicines. http://www. agenziafarmaco.gov.it/allegati/det_20marzo2008. pdf. Accessed 14 Oct 2019.

10. Smolen JS, Landewe R, Bijlsma J, et al. EULAR recommendations for the management of rheumatoid arthritis with synthetic and biological diseasemodifying antirheumatic drugs: 2016 update. Ann Rheum Dis. 2017;76:960-77.

11. Fakhouri W, Lopez-Romero P, Antonelli S, et al. Treatment patterns, health care resource utilization and costs of rheumatoid arthritis patients in Italy: findings from a retrospective administrative database analysis. Open Access Rheumatol. 2018;10: 103-11.

12. Steffen A, Holstiege J, Klimke K, Akmatov MK, Batzing J. Patterns of the initiation of diseasemodifying antirheumatic drugs in incident rheumatoid arthritis: a German perspective based on nationwide ambulatory drug prescription data. Rheumatol Int. 2018;38:2111-20.

13. Doria A, Zavaglia D. Monotherapy is a relevant option in rheumatoid arthritis treatment: a literature review. Clin Exp Rheumatol. 2019;37:862-71.

14. Stamm TA, Reichardt B, Zwerina J, Ritschl V, NellDuxneuner V. Use of biological disease modifying antirheumatic drugs in rheumatoid arthritis in Austria from 2008 to 2011: a retrospective analysis of $72 \%$ of the population. Wien Klin Wochenschr. 2018;130:230-7. 
15. Taylor PC. Clinical efficacy of launched JAK inhibitors in rheumatoid arthritis. Rheumatology (Oxford). 2019;58:i17-26.

16. Matsuno H, Atsumi T, Takei S, et al. Safety of baricitinib (Bari) under clinical settings in patients (pts) with rheumatoid arthritis (RA), using data from all-case post-marketing surveillance and spontaneous reports. In: The 63rd Annual Scientific Meeting of the Japan College of Rheumatology, Kyoto, Japan, Japan College of Rheumatology; 2019, p. S291.

17. Meissner Y, Kekow J, Klopsch T, Kühne C, Zink A, Strangfeld A. Erste erfahrungen mit baricitinib aus dem rheumatologischen alltag. In: 46. Kongress der Deutschen Gesellschaft für Rheumatologie (DGRh), 32. Jahrestagung der Deutschen Gesellschaft für Orthopädische Rheumatologie (DGORh), Wissenschaftliche Herbsttagung der Gesellschaft für
Kinder- und Jugendrheumatologie (GKJR). Mannheim: German Medical Science; 2019.

18. Guidelli GM, Generali E, Bazzani C, et al. AB0446 real-life use of baricitinib in rheumatoid arthritis: a multicenter observational study of 150 patients. Ann Rheum Dis. 2019;78:1685-6.

19. Page J, Kearsley-Fleet L, Davies R, Watson K, Hyrich K, Lunt M. AB0434 early experience with JAK inhibitor prescribing in the UK: results from the british society for rheumatology biologics register for rheumatoid arthritis (BSRBR-RA). Ann Rheum Dis. 2019;78:1680.

20. Lwin MN, Cole Z, Holroyd C, et al. E062 Baseline characteristics of patients with rheumatoid arthritis starting the janus kinase inhibitor baricitinib: reallife observation study. Rheumatology. 2019. https://doi.org/10.1093/rheumatology/kez110.060. 